\title{
Molecular Simulation of Phase Equilibria FOR COMPLEX FLUIDS
}

\author{
Final Report \\ DOE Project \# DE-FG02-89ER14014 \\ Division of Chemical Sciences \\ Office of Basic Energy Sciences \\ February 10, 1999 \\ Principal Investigator \\ Athanassios Z. Panagiotopoulos \\ Institute for Physical Science and Technology and \\ Department of Chemical Engineering, \\ University of Maryland, College Park, MD 20742-5201
}

\section{TABLE OF CONTENTS}

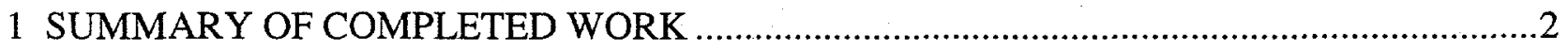

1.1 Development of computational methodologies............................................

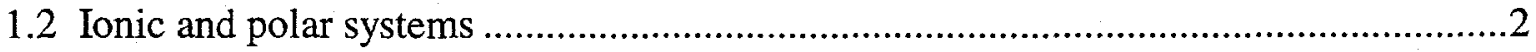

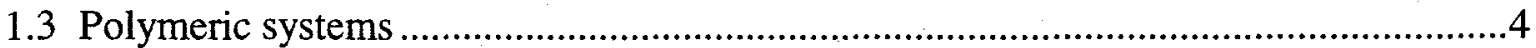

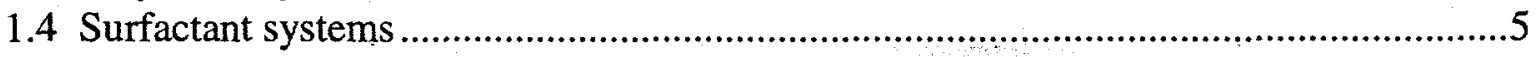

2 AWARDS, PUBLICATIONS AND PRESENTATIONS (JUNE. ‘95 - NOVEMBER ‘98) .......6

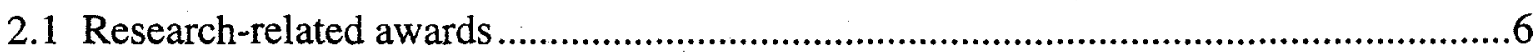

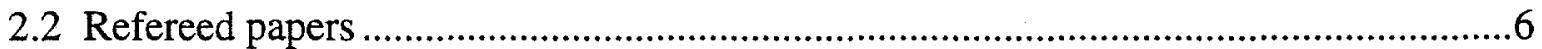

2.3 Conference presentations ..................................................................................

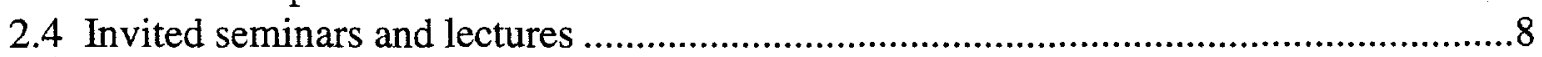

3 PERSONNEL ASSOCIATED WITH THE PROJECT ….................................................11 


\section{DISCLAIMER}

This report was prepared as an account of work sponsored by an agency of the United States Government. Neither the United States Government nor any agency thereof, nor any of their employees, make any warranty, express or implied, or assumes any legal liability or responsibility for the accuracy, completeness, or usefulness of any information, apparatus, product, or process disclosed, or represents that its use would not infringe privately owned rights. Reference herein to any specific commercial product, process, or service by trade name, trademark, manufacturer, or otherwise does not necessarily constitute or imply its endorsement, recommendation, or favoring by the United States Government or any agency thereof. The views and opinions of authors expressed herein do not necessarily state or reflect those of the United States Government or any agency thereof. 


\section{DISCLAIMER}

Portions of this document may be illegible in electronic image products. Images are produced from the best available original document. 


\section{SUMMARY OF COMPLETED WORK}

The general area of this project is the development and application of molecular simulation methods for prediction of equilibrium properties of complex fluids. In earlier project periods, we have developed the Gibbs ensemble methodology for direct determination of phase equilibria. The technique has met with considerable success and is now in widespread use throughout the world. In the most recent project period, we focused on polar/ionic, polymeric, and surfactant systems. We have made considerable progress in developing novel algorithms to meet the computational challenges presented by the strong interactions in these systems, and have generated data for well-defined models that can be used to test theories and compare to experimental data. In the following sections we describe progress in each of the focus areas of the project, with references [in square brackets] to the accompanying list of publications [\$ 2.2] for additional technical details.

\subsection{Development of Computational Methodologies}

The main methodological developments in the current reporting period were the combination of thermodynamic scaling ideas with the Gibbs ensemble [ref. 7 in $\$ 2.2$ ], and the application of histogram reweighting and mixed-field finite-size scaling concepts to pure fluids [10], mixtures [13] and surfactant solutions [14]. Histogram reweighting methods ${ }^{1}$ hold significant promise for accurate determination of equilibrium properties over a broad range of conditions. Until recently, these methods had been applied primarily to spin lattice models. The techniques are based on performing a small number of grand canonical Monte Carlo simulations and combining the results to obtain the system free energy as a function of density and temperature. In addition to phase equilibria, volumetric properties and even derivative properties such as second virial coefficients can be obtained. When coupled with mixed-field finite-size scaling concepts ${ }^{2}$ they also provide estimates of critical parameters. For example, in [13], we obtained the critical parameters of the full (untruncated) Lennard-Jones potential as $T_{\mathrm{c}}=1.3120 \pm 0.0007, \rho_{\mathrm{c}}=0.316$ \pm 0.001 and $P_{\mathrm{c}}=0.1279 \pm 0.0006$. The uncertainties in the critical properties are an order of magnitude smaller than for previous calculations. In our work over the current reporting period, we have demonstrated the generality and usefulness of histogram-reweighting techniques for complex fluids and resolved a number of methodological issues related to their application to realistic systems.

\subsection{Ionic and polar systems}

Electrolyte solutions, molten salts, and certain colloidal systems are all examples of systems in which the dominant interparticle forces are coulombic interactions. Because of the long-range nature of the coulombic forces, accurate statistical-mechanical descriptions of these systems are difficult to obtain, especially at low temperatures.

${ }^{1}$ Ferrenberg, A.M. and R.H. Swendsen, "Optimized Monte Carlo Data Analysis," Phys. Rev. Lett. 63, 1195-98 (1989).

${ }^{2}$ Nigel B Wilding, Simulation studies of fluid critical behaviour, J. Phys.: Condens. Matter 9, 585-612 (1997). 
There have been several simulation and theoretical studies of the simplest possible model for electrolyte solutions, the "restricted primitive model" (RPM) which consists of charged hard spheres of equal diameter, half positively and half negatively charged, in a structureless dielectric continuum. Work completed in previous project periods ${ }^{3}$ produced estimates of the coexistence curve and critical parameters of this model that facilitated the development of statistical mechanical theories for ionic fluids. ${ }^{4,5}$ In a new study of this system [ref. 12 in $\S 2.2$ ] we demonstrated that the most accurate computational methods currently available, namely histogram-reweighting methods combined with finite-size scaling, result in critical parameters significantly shifted relative to earlier Gibbsensemble based calculations. Figure 1 illustrates the dependence of the critical parameters on system size from our work and compares our results to the independent study of Caillol et $a l^{6}{ }^{6}$ performed on a four-dimensional hypersphere. The new estimate of the critical temperature $\left(T_{c}=0.0490 \pm 0.0003\right)$ is ten percent lower than the earlier Gibbs-ensemble based estimate, primarily due to the neglect of systematic corrections for finite-size effects near the critical point. The critical density estimate $\left(p_{c}=0.070 \pm 0.005\right)$ is higher than our earlier estimate because of the high asymmetry of the RPM phase diagram and the earlier overestimate of the critical temperature. In our recent work, we have also obtained the first estimate of
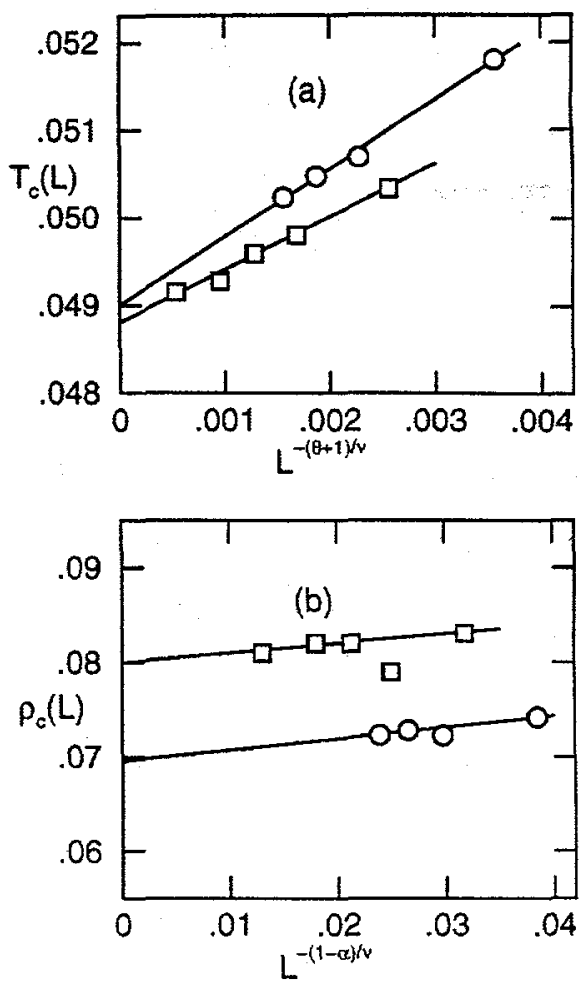

Fig. 1. Scaling plots for the critical temperature (top) and density (bottom ) for the RPM. Squares are from the study of Caillol et al. and circles from our work [12].

the critical pressure and the critical compressibility factor. We have obtained $Z_{c}=0.024 \pm 0.004$, an order of magnitude lower than for nonionic fluids.

Early in the present period, we completed our study of the two-dimensional analog of the RPM using thermodynamic scaling methods [6]. The system exhibits a continuous conductor/insulator transition at low densities and high temperatures in addition to the first-order gas/liquid transition at lower temperatures.

In mixtures of ionic and non-ionic components (e.g. aqueous electrolyte solutions), solvent polarizability effects are important. Polarizable Stockmayer model fluids [8] and polarizable models for water [11] were studied in the present reporting period. Currently

${ }^{3}$ G. Orkoulas and A.Z. Panagiotopoulos, "Free energy and phase equilibria for the restricted primitive model of ionic fluids from Monte Carlo Simulations," J. Chem. Phys. 101, 1452-59 (1994) - cited 59 times as of 11/98.

${ }^{4}$ G. Stell, "Criticality and phase-transitions in ionic fluids," J. Stat. Phys., 78, 197-238 (1995).

5 M.E. Fisher, "The nature of criticality in ionic fluids," J. Phys.: Condens. Matter 8, 9103-09 (1996).

${ }^{6}$ Caillol, J. M.; Levesque, D.; Weis, J. J., "A Monte Carlo finite size scaling study of charged hard-sphere criticality," J. Chem. Phys. 107, 1565-75 (1997). 
available polarizable water models do not represent accurately the measured phase behavior of water in the vicinity of the critical region. This limits their usefulness for molecular simulations of electrolyte/water mixtures at elevated temperatures and pressures.

\subsection{Polymeric systems}

\section{Continuous-space systems}

Our investigations of mixing properties and free energies of model polymer/solvent systems [references 1 and 2 in $\S 2.2$ ] were published in the present reporting period. The chain increment method $^{7}$ developed with DOE support in a previous project period, was used to obtain the chemical potential as a function of chain length, temperature and density by summing all contributions for chains of length less than the target chain. We also completed a study of the effect of chain flexibility on the phase behavior of homopolymers [ref. 4]. The surprising result from this study is that more flexible chains (which have fewer intermolecular contacts) are nevertheless less volatile and have a critical temperature higher than for stiffer chains.

\section{$\underline{\text { Lattice systems }}$}

Histogram-reweighting and finite-size scaling methods were used to obtain phase diagrams and critical parameters of lattice homopolymers of much greater length (up to 1000 monomers) than previously possible. The equivalence of the three possible definitions of the $\Theta$ temperature was established to a high accuracy. The three possible definitions are (a) the limit of the critical temperature for infinite chains, (b) the temperature at which the chain-chain second virial coefficient vanishes and (c) the temperature at which chain dimensions behave quasi-ideally. The scaling of the critical temperature with chain length was found to agree with simple mean-field theory predictions, but the scaling of critical density was found to be in disagreement with most theoretical predictions but in agreement with experimental measurements on polymer-solvent systems. In a recent independent study, which also used histogram reweighting methods, ${ }^{8}$ it has been suggested that logarithmic corrections to scaling may be masking the asymptotic scaling of the critical density with chain

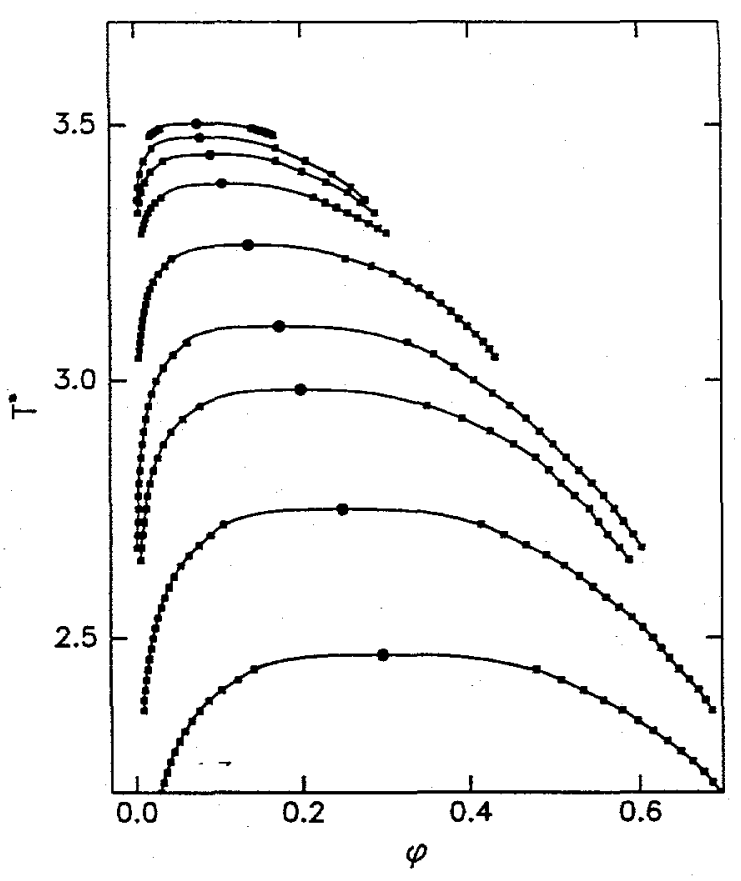

Fig. 2 [ref. 10]. Phase diagrams for simple cubic lattice homopolymers of length $1000,800,600,400,200,100,64$, 32 and 16 monomers (from top to bottom).

${ }^{7}$ Kumar, S.K.; Szleifer, I.; Panagiotopoulos, A.Z, "Determination of the chemical potentials of polymeric systems from Monte Carlo Simulations," Phys. Rev. Lett. 66, 2935-8 (1991).

8 Frauenkrom,
(1997). 
length. In order to decide this question, future studies for significantly longer chain lengths will need to be undertaken.

\subsection{Surfactant systems}

The final focus area of this project is surfactant solutions. The surfactant models we use are short diblock copolymers immersed in a solvent consisting of a binary mixture of monomeric beads. One of the solvent species preferentially interacts with the "head" block of the surfactant, thus being an analog of water in real ternary surfactant mixtures, while the other solvent species is attracted to the "tail" surfactant block and represents oil. Unfavorable interactions between the two solvent species lead to immiscibility between "water" and "oil." Our goals in this area are to obtain complete information on both phase behavior and formation of micelles or other microstructures.

Our early work [references 5 and 9] was based on Gibbs ensemble simulations to determine the phase behavior and large-scale canonical (constant- $N V T$ ) simulations to obtain information on the formation of aggregates. We found that the canonical-ensemble calculations suffered from hysteresis effects at lower temperatures, making it impossible to obtain reliable information on the aggregate size distributions. The difficulty is the result of the large free-energy cost of breaking up a micellar aggregate. In our most recent work, significant advancements in methodologies for studying self-organizing systems have been achieved [14]. Histogram-reweighting grand canonical simulations in relatively small systems were shown to give reliable information on the critical micelle concentration (CMC) and aggregate size distributions for these systems. In Figure 3, the osmotic pressure curves for a model surfactant at two different temperatures are shown. As is the case for experimental data for micelle-forming solutions, a clear break is present at a concentration that can be identified as the CMC. In reference [14], we studied the effects of temperature and surfactant architecture on the micellization behavior of two model surfactants, $H_{4} T_{4}$ and $H_{3} T_{3}$. Calculated cmc values for the two symmetric surfactants studied in [14] are shown in Fig. 4. The fitted lines to the curves are of the form $\ln \left(\varphi_{\mathrm{cmc}}\right)=A+B / T$. Parameter $B$ is proportional to the enthalpy of micellization, assuming that the system is ideal at the cmc. The micellization enthalpies for $\mathrm{H}_{3} \mathrm{~T}_{3}$ and $\mathrm{H}_{4} \mathrm{~T}_{4}$ were found to be in a ratio of exactly $3: 4$, the same as the ratio of chain lengths for the two surfactants.

A typical configuration of $\mathrm{H}_{4} \mathrm{~T}_{4}$ surfactant at conditions for which several micelles are present in the system is shown in Figure 3. Because of the periodic boundary conditions, several 


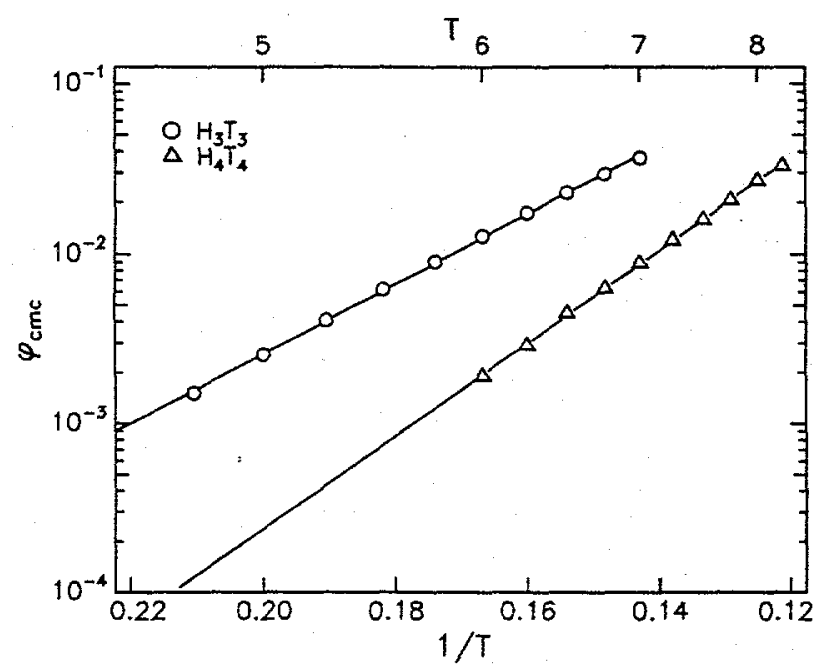

Fig. 4. Dependence of the CMC on temperature and surfactant architecture [ref. 14].

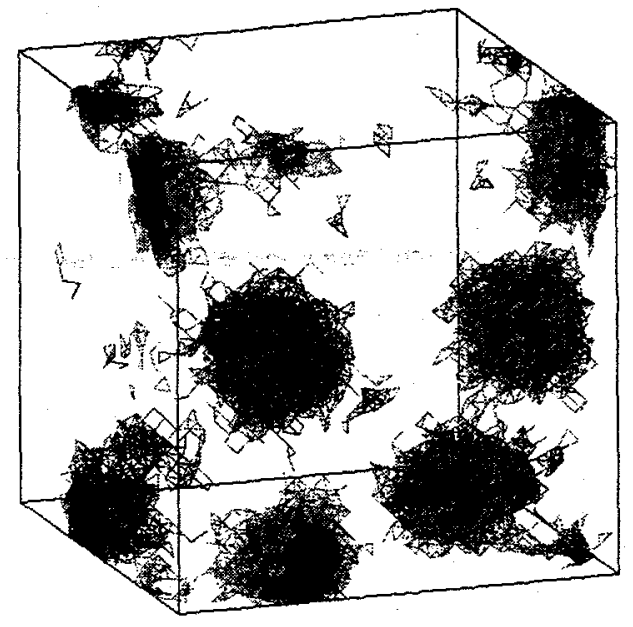

Fig. 5. A snapshot of an instantaneous configuration in the $H_{4} T_{4}$ system.

aggregates appear in cross-section, revealing the densely packed hydrophobic cores of the micelles.

\section{AwARDs, PUBliCATIONS AND PRESENTATIONS RESUlTING FROM THIS PROJECT (JUNE. '95 - NOVEMBER '98)}

\subsection{Research-related Awards}

In the course of the present project, the PI received two major research-related awards. The first was the Colburn Award of the American Institute of Chemical Engineers awarded in November 1995 "for excellence in publications on the part of a younger member of the American Institute for Chemical Engineers." The Colburn award is given annually to a single researcher under 35 years of age after a highly competitive nomination and selection process. In May 1998 the PI received the inaugural J.M. Prausnitz award "for outstanding achievements in applied chemical thermodynamics." The award is connected to the triennial series of international conferences on fluid properties and phase equilibria.

\subsection{Refereed papers}

1. Y.-J: Sheng, A. Z. Panagiotopoulos and D.P. Tassios, "Activity coefficients in nearly athermal model polymer/solvent systems," AIChE J., 41, 2306-13 (1995).

2. Y.-J. Sheng, A.Z. Panagiotopoulos and S.K. Kumar, "Mixing Properties of Model Polymer/ Solvent Systems," J. Chem. Phys., 103, 10315-24 (1995).

3. A.Z. Panagiotopoulos, "Current Advances in Monte Carlo Methods," Fluid Phase Equil., 116, 257-266 (1996). 
4. Y.-J. Sheng, A.Z. Panagiotopoulos and S.K. Kumar, "Effect of Chain Stiffness on Polymer Phase Behavior," Macromolecules, 29, 4444-6 (1996).

5. A.D. Mackie, K. Onur and A.Z. Panagiotopoulos, "Phase Equilibria of a lattice model for an oil-water-amphiphile mixture," J. Chem. Phys., 104, 3718-25 (1996).

6. G. Orkoulas and A.Z. Panagiotopoulos, "Phase Diagram of the Two-Dimensional Coulomb Gas: A Thermodynamic Scaling Monte Carlo Study," J. Chem. Phys., 104, 7205-9 (1996).

7. K. Kiyohara, T. Spyriouni, K. E. Gubbins and A. Z. Panagiotopoulos, "ThermodynamicScaling Gibbs Ensemble Monte Carlo: A new method for determination of phase coexistence properties of fluids," Molec. Phys., 89, 965-74 (1996).

8. K. Kiyohara, K. E. Gubbins and A. Z. Panagiotopoulos, "Phase Coexistence Properties of Polarizable Stockmayer Fluids," J. Chem. Phys., 106, 3338-47 (1997).

9. D. Mackie, A. Z. Panagiotopoulos and I. Szleifer, "Aggregation Behavior of a Lattice Model for Amphiphiles," Langmuir, 13, 5022-31 (1997).

10. A.Z. Panagiotopoulos, V. Wong and M.A. Floriano, "Phase Equilibria of Lattice Polymers from Histogram Reweighting Monte Carlo Simulations," Macromolecules, 31, 912-918 (1998).

11. K. Kiyohara, K.E. Gubbins and A.Z. Panagiotopoulos, "Phase Coexistence Properties of Polarizable Water Models," Molec. Phys., 94, 803-8 (1998).

12. G. Orkoulas and A.Z. Panagiotopoulos, "Phase Behavior of the Restricted Primitive Model and Square-Well Fluids from Monte Carlo Simulations in the Grand Canonical Ensemble," J. Chem. Phys., 110, 1581-90 (1998).

13. J.J. Potoff and A.Z. Panagiotopoulos, "Critical Point and Phase Behavior of a LennardJones Mixture from Histogram Reweighting Monte Carlo Simulations," J. Chem. Phys., 109, 10914-20 (1998).

14. M.A. Floriano, E. Caponetti and A.Z. Panagiotopoulos, "Micellization in Model Surfactant Systems," Langmuir, submitted for publication (1998).

\subsection{Conference presentations}

1. "Ionic Phase Transitions in Two and Three Dimensions from Monte Carlo Simulations," paper 55d, Annual AIChE meeting, Miami, FL, Nov. 12-17, 1995 (with G. Orkoulas).

2. "Statistical Thermodynamic Theory and Molecular Simulations of Polymers Confined in Narrow Pores," paper 48g, Annual AIChE meeting, Miami, FL, Nov. 12-17, 1995 (with L. Vega, M. Carignano and I. Szleifer).

3. "Phase Behavior and Micellization of a Model Amphiphile System through Monte Carlo Simulation," paper 96b, Annual AIChE meeting, Miami, FL, Nov. 12-17, 1995 (with A. Mackie and K. Onur). 
4. "Monte Carlo Simulation of Phase Behavior and Micellization for Model Amphiphile Systems," invited paper at the ACS National Meeting, New Orleans, LA, March 24-28, 1996 (with A. Mackie, and K. Onur).

5. "Monte Carlo Methods for Predictions of Phase Transitions in Fluids," invited paper at the ACS National Meeting, New Orleans, LA, March 24-28, 1996.

6. "Monte Carlo Simulations of Stockmayer Fluids," paper 13k, Annual AIChE meeting, Chicago, IL, Nov. 10-15, 1996 (with K. Kiyohara and K. Gubbins).

7. "Molecular Modeling of Phase Equilibria," paper 199d, Annual AIChE meeting, Chicago, IL, Nov. 10-15, 1996 (with V. Harismiadis, J. Vorholz and J. Errington).

8. "Finite-size Scaling and Approach to Criticality in Monte Carlo Simulations," paper 201a, Annual AIChE meeting, Chicago, IL, Nov. 10-15, 1996 (with G. Orkoulas and J. Potoff).

9. "Phase Coexistence Properties of Polarizable Water Models," paper presented at the $2 \mathrm{~d}$ Molecular Modeling and Molecular Simulation Conference, Tokyo, Japan, Jan. 12-15, 1997 (with K. Kiyohara and K. Gubbins).

10. "Critical Point and Coexistence Curve Properties for Ionic and Dipolar Fluids," presented at the $13^{\text {th }}$ Symposium on Thermophysical Properties, Boulder, CO, June 22-27, 1997 (with G. Orkoulas and J. Potoff).

11. "Phase Behavior of Lattice Polymers and Surfactants by Histogram Reweighting Monte Carlo Simulations," presented at the International Conference on the Morphology and Kinetics of Phase Separating Complex Fluids, Messina, Italy, June 24-28, 1997 (with V. Wong, M.A. Floriano and E. Caponetti).

12. "Critical Properties and Phase Coexistence in Polymer Solutions by HistogramReweighting and Finite-Size Scaling Monte Carlo Methods" paper 161j, Annual AIChE Meeting, Los Angeles, CA, Nov. 16-21, 1997 (with V. Wong).

13. "Computer Simulation of Phase Equilibria of Polyampholyte Systems," paper W11.04, March APS Meeting, Los Angeles, CA March 16-20, 1998 (with R. Ballamudi and S. Kumar).

14. "Critical Curves and Phase Behavior of Mixtures from Histogram Reweighting Monte Carlo Simulations," paper 72f, Annual AIChE meeting, Miami Beach, FL, November 1620, 1998 (with J.J. Potoff).

\subsection{Invited seminars and lectures}

1. "Fundamentals of Monte Carlo Simulation," Invited lecture at the 7th International Conference on Fluid Properties and Phase Equilibria for Chemical Process Design," Snowmass, CO, June 18-23, 1995.

2. "Molecular Simulation of Ionic and Polymer Systems," Los Alamos National Laboratory, June 27, 1995.

3. "Molecular Simulation of Phase Equilibria for Ionic and Polymeric Fluids," Invited paper at the 4th Asian Thermophysical Properties Conference, Tokyo, Japan, Sept. 5-8, 1995. 
4. "Molecular Simulation of Phase Equilibria for Ionic, Polymeric and Surfactant Systems," Department of Chemical Engineering, Univ. of Tennessee, Sept. 19, 1995.

5. "Molecular Simulation of Phase Equilibria for Polymeric and Surfactant Systems," Department of Chemical Engineering, Univ. of Missouri-Rolla, Sept. 20, 1995.

6. "Molecular Simulation: A new tool for engineers," van Ness lecture, Department of Chemical Engineering, Rennselaer Polytechnic Institute, Oct. 11, 1995.

7. "Phase Equilibria for Polymeric and Surfactant Systems," van Ness lecture, Department of Chemical Engineering, Rennselaer Polytechnic Institute, Oct. 12, 1995.

8. "Monte Carlo Simulations of Polymeric and Surfactant Systems," Department of Chemical Engineering, Univ. of Texas at Austin, Oct 17, 1995.

9. "Molecular Simulation of Phase Equilibria," Department of Chemical Engineering, Univ. of California, Berkeley, Dec. 4, 1995.

10. "Molecular Simulation of Phase Transitions," Demokritos National Research Center for the Physical Sciences, Athens, Greece, Dec. 27, 1995.

11. "Simulations of Phase Transitions in Ionic and Surfactant Systems," Invited lecture at the Workshop on Current Problems in Complex Fluids, Oaxaca, Mexico, Jan. 3-7, 1996.

12. "Novel methods for Monte Carlo Simulations of Phase Transitions for Fluids"' Invited lecture at the XXV Statistical Physics Conference, Cuernavaca, Mexico, Jan. 10, 1996.

13. "Molecular Simulation of Phase Equilibria," Department of Chemical Engineering, Northwestern University, Jan. 25, 1996.

14. "Molecular Simulation of Phase Equilibria," Phillips Petroleum Company, Bartlesville, Oklahoma, Feb. 16, 1996.

15. "Molecular Simulation of Phase Equilibria," Department of Chemical Engineering, Univ. of California, Santa Barbara, Apr. 4, 1996.

16. "Molecular Simulation of Phase Transitions," Department of Chemical Engineering, City University of New York, Apr. 15, 1996.

17. "Molecular Simulation of Phase Transitions," Thiele Lecture, Department of Chemical Engineering, Univ. of Notre Dame, Oct. 1, 1996.

18. "Molecular Simulation of Phase Transitions in Complex Fluids," Department of Chemical Engineering, Univ. of Southern California, Nov. 20, 1996.

19. “Molecular Simulation of Phase Transitions in Complex Fluids," Department of Chemical Engineering, California Institute of Technology, Nov. 21, 1996.

20. "Phase Transitions in Polymeric and Ionic Systems," National Research Center "Demokritos," Athens, Greece, Dec. 23. 1996.

21. "Simulation of Phase Equilibria in Complex Fluids," invited lecture, 2d Molecular Modeling and Molecular Simulation Conference, Tokyo, Japan, Jan. 12-15, 1997.

22. "Monte Carlo Simulation of Phase Equilibria," Department of Chemical Engineering, University of California, Los Angeles, Jan. 31, 1997. 
23. "Molecular Simulation of Phase Transitions in Complex Fluids," Institute of Physical Science and Technology and Dept. of Chemical Engineering, University of Maryland, College Park, Feb. 11, 1997.

24. "Molecular Simulation of Phase Transitions in Fluids," Invited talk at the John Valleau 65th Birthday Symposium, Toronto, Canada, April 18, 1997.

25. "Molecular Simulation of Phase Transitions," Department of Chemical Engineering, Univ. of Delaware, Sept. 12, 1997.

26. "Chance and Necessity: Simulating Phase Transitions by Monte Carlo Methods," Department of Physical Chemistry, Univ. of Palermo, Italy, Oct. 14, 1997.

27. "Molecular Simulation of Phase Transitions in Ionic, Polymeric and Surfactant Systems," Department of Physical Chemistry, Univ. of Palermo, Italy, Oct. 15, 1997.

28. "Computational Molecular Simulation and Potentials" invited lecture at the NSF workshop in "Future Directions in Molecular Modeling and Simulation," Arlington, VA, Nov. 3-5, 1997.

29. "Monte Carlo Simulations of Phase and Conformational Transitions in Polymeric Systems," invited lecture at the ACS Workshop on Molecular Modeling of Polymers, Isle of Palms, S. Carolina, March 1-3, 1998.

30. "Monte Carlo Simulations of Phase Trasitions in Fluids," invited lecture at the APS meeting in Los Angeles, CA, March 15-20, 1998.

31. "Molecular Simulation of Phase Equilibria for Fluid Mixtures," invited lecture at the IMP workshop on Computer Simulations in Oil Problems, Mexico City, Mexico, Apr. 6-8, 1998.

32. "John M. Prausnitz Award Lecture" at the International Conference on Fluid Properties and Phase Equilibria for Process and Product Design, Nordwijkerhout, The Netherlands, April 30, 1998.

33. "Phase and Conformational Transitions in Model Surfactant Systems: Monte Carlo Simulations and Mean-Field Theory," IPST Statistical Physics Seminar May 5, 1998.

34. "Recent Development in MC Methods for Phase Transitions in Fluids" invited lecture at the $5^{\text {th }}$ Liblice Conference on the Statistical Mechanics of Liquids, Zelezna Ruda, Czech Republic, June 7-12, 1998.

35. "Phase Transitions in Model Amphiphile Systems: Simulations and Mean-Field Theory" three invited lectures at the NATO Advanced Study Insitute on Computer Simulation of Liquid Crystals, Erice, Italy, June 11-21, 1998.

36. "Molecular Simulation of Phase Equilibria" invited lecture, Shell E\&P Technology Company Seminar, Houston, TX, June 25, 1998.

37. "Molecular Simulation of Phase Transitions," Department of Chemical Engineering, University of Maine, Orono, Maine, October 16, 1998.

38. "Micellization in Model Surfactant Systems," National Institute for Standards and Technology, October 21, 1998. 
39. "Molecular and Mesoscopic Simulations of Phase Equilibria," invited lecture at the J. M. Prausnitz Symposium, AIChE Annual Meeting, Miami Beach, Florida, November 16, 1998.

40. "Advances in Molecular Simulations: A Review," invited lecture at the Plenary Session on Advances in Molecular and Materials Modeling, AIChE Annual Meeting, Miami Beach, Florida, November 16, 1998.

\section{PERSONNEl AsSOCIATED WITH THE PROJECT}

The following researchers have been associated with the present project in the period July 1995 to November 1998.

A. Principal Investigator: Athanassios Z. Panagiotopoulos

B. Post-doctoral Associates

Avik Chatterjee (Ph.D in Chemistry, Cornell U., 1996). Dr. Chatterjee joined our group at Maryland in September 1998. He previously worked with Prof. Loring at Cornell and Prof. Schweizer at Illinois. His current project focuses on micellization in model surfactant systems.

Gerassimos Orkoulas (Ph.D, Cornell U., 1997). Dr. Orkoulas came to Maryland in May 1998. He is jointly supervised here by Prof. M. Fisher and the PI, and is working on critical behavior of ionic fluids.

C. Graduate Students

Allan Mackie (B.S., U. of Edinburgh, 1989). Mr. Mackie received his Ph.D. in August of 1995. His thesis was on molecular simulation of homopolymers and surfactant solutions. He is presently an Assistant Professor at the Univ. of Tarragona, Spain.

Gerassimos Orkoulas (B.S., U. of Patras, 1989). Mr. Orkoulas' doctoral thesis project was on phase equilibria of ionic systems $\mathrm{He}$ received his $\mathrm{PhD}$ in September 1997. $\mathrm{He}$ is currently a post-doctoral fellow at the Univ. of Maryland (see above).

Y.-Jane Sheng (B.S. and M.S., National Taiwan University, 1990). Ms. Sheng completed her Ph.D. in August 1995. Her thesis was on mixing properties and phase equilibria of polymeric systems. She is presently an Assistant Professor at National Taiwan Univ.

Jeff Potoff (B.S. Michigan State Univ., 1994). Mr. Potoff joined our group in January 1995. His research focuses on polar fluids and development of computational methodologies.

C. Undergraduate Students

Paul Barranelo

Andrew Schultz

Pasut Ratanabanangkoon 